\title{
Corela
}

Cognition, représentation, langage

HS-1 | 2005

Colloque AFLS

\section{L'expression de la conjecture : une analyse contrastive français/berbère (kabyle)}

\author{
Linda Bellahsène
}

\section{(2) OpenEdition}

Journals

\section{Édition électronique}

URL : http://journals.openedition.org/corela/1097

DOI : 10.4000/corela.1097

ISSN : 1638-573X

Éditeur

Cercle linguistique du Centre et de l'Ouest - CerLICO

\section{Référence électronique}

Linda Bellahsène, «L'expression de la conjecture : une analyse contrastive français/berbère (kabyle) », Corela [En ligne], HS-1 | 2005, mis en ligne le 16 février 2005, consulté le 19 avril 2019. URL : http:// journals.openedition.org/corela/1097 ; DOI : 10.4000/corela.1097

Ce document a été généré automatiquement le 19 avril 2019

\section{(c) (i) (2)(2)}

Corela - cognition, représentation, langage est mis à disposition selon les termes de la licence Creative Commons Attribution - Pas d'Utilisation Commerciale - Partage dans les Mêmes Conditions 4.0 International. 


\title{
L'expression de la conjecture : une analyse contrastive français/berbère (kabyle)
}

\author{
Linda Bellahsène
}

\section{Introduction ${ }^{1}$}

$1 \quad$ L'objet de cet article est de proposer une analyse contrastive de la particule préverbale [ad + ili (aoriste)] suivi du thème de l'aoriste intensif ou du prétérit en berbère Kabyle et $\mathrm{du}$ futur conjectural en français. Comme cadre d'analyse, nous utiliserons le modèle calculatoire et cognitif du temps et de l'aspect de L. Gosselin $(1996,2000)$. Nous limiterons notre analyse $\mathrm{e}^{2}$ aux contextes où le futur conjectural porte sur une situation passée exprimée en français par un futur antérieur et en berbère kabyle par l'auxiliaire « temporel » ili précédé de la particule préverbale ad.

2 Wilmet atteste les premiers emplois du futur conjectural en moyen français. Dans un énoncé au futur conjectural, «le locuteur postpose son jugement, enregistre l'événement antérieur sous bénéfice d'inventaire » (1997 : 380). Il paraphrase l'énoncé par : «la vérité d'aujourd'hui n'apparaîtra en pleine lumière que demain » (ibidem). En français, le futur conjectural référant à une situation présente est limité aux seuls verbes auxiliaires avoir et être dans des structures à présentatif ${ }^{3}$. Ces restrictions d'emploi en ont modifiées l'usage ; la forme en ça/ce doit être ou le futur conjectural référant à une situation passée lui sont préférés. Les différentes formes du futur ont pour fonction de situer le procès après le moment de l'énonciation. Dans cet emploi, le futur réfère à une situation présente ou passée dans le cas du futur antérieur, que nous illustrons par les exemples suivants :

(1) Cocarel : (...) Comment, ma femme a déjà pris son bain? Oh! Elle aura changé

d'idée. (Feydeau, Un bain de ménage, Acte I, scène VIII) 
(2a) Comment diable est-ce dans mes bottes? Ce sera, sans doute, répondit-elle, tombé du vieux carton aux factures, qui est sur le bord de la planche. (Flaubert, Madame Bovary, p. 406-407)

3 En kabyle, le verbe ili à l'aoriste (être, exister) précédé de la particule préverbale ad peut être utilisé comme auxiliaire temporel (Naït-Zerrad, 2001). Il est considéré comme marquant l'expression de la probabilité ou de l'antériorité dans le futur s'il est suivi du thème du prétérit (3) ou encore comme l'expression d'une probabilité ${ }^{4}$ ou d'un duratif ou itératif dans le futur s'il est suivi du thème de l'aoriste intensif (4).

(3) Ad yili yeffey. [ad + (ili à l'aoriste) + prétérit]

Il doit être sorti/il sera sorti.

(4) Ad yili ixeddem. [ad + (ili à l'aoriste) + aoriste intensif]

Il doit être en train de travailler/il sera en train de travailler. (Exemples cités par

Naït-Zerrad, $2001: 116-117)$

Dans notre analyse, nous essaierons de montrer que le processus inférentiel mis en œuvre engendre une valeur de probabilité/plausibilité impliquant la non assertion de la proposition. Ce temps de dicto (Martin, 1987: 111) est le temps de la prise en charge différée; le futur conjectural porte donc sur la vérification de cette hypothèse comme modalité d'assertion (Gosselin, 1999: 38) et non sur la proposition, d'où la remarque de Schäfer-Prieß :

Cette séparation de la proposition et de sa vérification distingue le futur de probabilité du futur dit temporel où la vérification est simultanée à l'événement, les deux dans l'avenir. $(2001,13)$

Partant de ce constat, nous montrerons que le futur conjectural exprime un méta-procès qui porte sur la vérification. Il s'agira donc d'une irrévocabilité rétrospective située dans le futur. Le futur conjectural sera alors considéré comme une modalité de dicto véridicible.

\section{Le rôle du contexte}

6 Traditionnellement, le futur conjectural est considéré comme un marqueur de modalité dont la valeur sémantique est décrite comme présentant un fait qui sera vérifié ou pris en charge dans l'avenir. Cette idée avait déjà été introduite par Damourette et Pichon (1911-1936), lorsque citant l'exemple suivant : « (...) Mais ce sera la fille à M. Pupin, disait Françoise qui préférait s'en tenir à une explication immédiate ", ils considéraient le futur conjectural comme situant l'événement à un moment où l'hypothèse explicative formulée par le locuteur est vérifiée. Autrement dit, le procès serait situé dans l'irrévocable et la vérification de celui-ci serait envisagée comme rétrospective. Néanmoins, cette notion de vérification ne peut s'appliquer à tous les énoncés au futur conjectural.

On remarque que l'hypothèse explicative exprimée dans le contexte énoncé en (1) peut être vérifiée tandis que le contexte de l'exemple (2a) ne le permet pas. Cette explication restera à l'état de supposition sauf si, par la suite, d'autres indices permettent d'invalider ou de valider le fait avancé. On pourra toujours imaginer une situation où le protagoniste découvre que l'objet en question ne pouvait pas, pour une raison $x$, se trouver dans le vieux carton aux factures.

8 Le même phénomène est observé dans l'interprétation d'un texte littéraire. Ainsi, si nous nous référons à l'analyse faite par Eco (1990), citant l'exemple de Zadig, les diverses énonciations visuelles auxquelles le protagoniste a affaire peuvent représenter une série 
décousue ou bien une séquence cohérente. Une série est reconnue comme une séquence textuelle quand on a trouvé le topic textuel qui permet d'établir une relation cohérente entre les divers indices textuels sans liens entre eux. Le lecteur doit émettre une série d'abductions dites hypocodées pour identifier un topic textuel. Comme le souligne Eco:

Très souvent, on ignore si le topic supposé est le «bon » et l'interprétation textuelle débouche sur des actualisations différentes et sémantiquement conflictuelles. Cela prouve que tout interprète d'un texte accomplit des abductions pour choisir entre les nombreuses lectures possibles de celui-ci. $(1990: 262)$

En considérant les deux exemples, nous constatons qu'ils s'inscrivent dans deux types de contexte différents, mettant chacun en œuvre un mécanisme inférentiel. Comme le précise Dendale :

Ils [devoir épistémique et futur conjectural] signalent tous les deux quelque chose à propos de la fiabilité de l'information (...) ou à propos de la façon dont le locuteur a obtenu son information - par observation directe, par emprunt à autrui, ou par inférence ou supposition - (...). $(2001,2)$

Dans l'exemple (1), le locuteur émet une hypothèse explicative, plausible, en inférant à partir de faits observés. Le protagoniste constate que sa femme a pris son bain; sachant qu'elle lui avait affirmé ne pas vouloir le prendre, il en tire la conclusion suivante : « Oh! Elle aura changé d'idée ». Cocarel ne cherche pas à formuler une loi universelle mais à désambiguïser un fait isolé. Cette explication, produit d'un raisonnement inférentiel, nécessite une vérification ou la révision éventuelle de sa croyance qu'il remet à plus tard en employant le futur antérieur. Dans l'exemple (2a), le contexte dans lequel le fait est observé ne permet pas de remettre la vérification de l'hypothèse explicative à plus tard. Nous avons là un objet supposé être tombé du carton et qui s'est retrouvé dans une paire de bottes. Cette action ne dépend pas d'un agent agissant qui serait susceptible d'être interrogé. Contrairement à ce qu'affirme Schrott :

Pour que l'emploi du futurE soit possible, il faut $\left(1^{\circ}\right)$ que la réalité décrite soit vérifiable (dans l'avenir) et $\left(2^{\circ}\right)$ que la supposition provienne du monde des expériences du locuteur et que la vérification de la supposition puisse se faire à partir de ce monde des expériences. (Cité par Dendale, 2001:8)

notre exemple (2a), bien qu'énoncé au futur conjectural, exprime une supposition qui ne sera pas vérifiable et qui provient du monde des expériences du locuteur ${ }^{5}$. De plus, il peut être remplacé par devoir.

(2b) Ce doit être, sans doute, répondit-elle, tombé du vieux carton aux factures, qui est sur le bord de la planche. (Flaubert, Madame Bovary, p. 406-407)

Nous avons donc deux types de contexte dans lesquels le futur conjectural se manifeste :

- un contexte d'ignorance ${ }^{6}$ permettant la vérification de l'hypothèse ;

- un contexte d'ignorance autorisant la supposition non vérifiable. Nous prendrons en considération uniquement les énoncés de contexte A.

\section{Le futur conjectural comme processus inférentiel}

12 Le raisonnement inférentiel est défini par Peirce (1955) comme un processus de la pensée qui permet de passer de ce que l'on sait (ou de ce que l'on croit savoir) à ce que l'on ne sait pas encore.

13 L'analyse sur les processus inférentiels mis en œuvre par le lecteur dans l'interprétation d'un texte littéraire (Eco, 1990) a montré qu'un fait singulier est pris comme hypothèse explicative d'un autre fait singulier, le premier fonctionnant à l'intérieur d'un univers 
textuel présenté comme une loi générale qui expliquerait le second ; ce qui nous conduit à émettre des conjectures sur des faits qui ne paraissent entretenir aucun lien entre eux. $\mathrm{Si}$ ces faits sont assertés sous la forme d'une hypothèse, c'est qu'ils sont généralement le résultat d'une interprétation conjecturale qui tend à être confirmée. Gärdenfors (1988) insiste sur le fait qu'une explication doit avant tout rendre une observation plus probable qu'elle n'est a priori.

Nous ne discuterons pas le type de raisonnement mis en œuvre par le locuteur pour la production de son hypothèse, nous renvoyons à l'analyse sur le modal devoir de Dendale \& De Mulder (1996) et Desclés \& Guentchéva (2001). Il a été remarqué que le futur conjectural met en œuvre un mécanisme inférentiel (entre autres, Vet \& Kampers-Manhe, 1995 ; Dendale, 2001). Vet \& Kampers-Manhe (1995) notent que la forme en -ra a pour fonction de signaler à l'interlocuteur que le locuteur a conclu à la vérité de la proposition par inférence. À partir de prémisses servant de fondement à son raisonnement, le locuteur produit une assertion dont la valeur de vérité reste à confirmer.

Les phrases au présent ou au passé précédant l'énoncé au futur conjectural produisent un univers référentiel (contexte) pour la construction d'un raisonnement inférentiel. Le futur conjectural construit une relation d'inférence à partir d'une situation effective présente. Cette relation d'inférence se fonde sur des faits observés dans le présent du locuteur. Dans ces exemples, nous pouvons remarquer que le locuteur prend connaissance d'une situation qui appelle une explication. Il émet une hypothèse qui tend à rendre compte de ces faits. En (5), l'hypothèse explicative fournit par le locuteur ne pourra pas être vérifiée (cf. Le rôle du contexte).

(5) Comment diable est-ce dans mes bottes ? Ce sera, sans doute, répondit-elle, tombé du vieux carton aux factures, qui est sur le bord de la planche. (Flaubert, Madame Bovary, p. 406-407)

(6) Cocarel: (...) Comment, ma femme a déjà pris son bain? Oh! Elle aura changé d'idée. (Feydeau, Un bain de ménage, Acte I, scène VIII)

De plus, dans les exemples (7a) et (7b), vous verrez a pour fonction d'affirmer qu'un procès énoncé sous le mode du probablement vrai se trouve déjà dans l'avenir au moment de l'énonciation. Les locuteurs des exemples (7a) et (7b) affirment qu'au moment de l'énonciation le domaine des faits à venir (domaine A chez Vet, 1993) contient respectivement le procès « être chez Mme Goupil » et « être tombé sur un spadassin ».

(7a) - Ah ! À moins de ça, disait ma tante. Il faudrait qu'elle soit venue pour les fêtes. C'est cela! Il n'y a pas besoin de chercher, elle sera venue pour les fêtes. Mais alors nous pourrions bien voir tout à l'heure Mme Sazerat venir sonner chez sa sœur pour le déjeuner. Ce sera ça! J'ai vu le petit de chez Galopin qui passait avec une tarte! Vous verrez que la tarte allait chez Mme Goupil.

(7b) Barillon, à part.- Vous verrez qu'avec ma veine ordinaire, je serai tombé sur un spadassin. (Haut à Flamèche) Il est fort aux armes, le maire ? (Georges Feydeau, Le mariage de Barillon, scène IX)

17 En interprétant l'exemple (8), le locuteur est amené à produire une inférence à partir de plusieurs hypothèses considérées comme des connaissances d'arrière-plan. Sachant que les billets cachetés d'une épingle sont des billets de femme (connaissance d'arrière-plan), il en déduit que c'est un billet doux (première hypothèse déduite) ; la proposition est au présent. Partant de cette conclusion, il va inférer qu'une fillette aura glissé le billet dans les mains du Comte.

(8) Le Comte (pendant qu'il parle, ainsi que Figaro, l'orchestre joue pianissimo.) Diantre soit des femmes, qui fourrent des épingles partout ! (Il la jette à terre, puis il lit le billet et le baise). 
Figaro qui a tout vu dit à sa mère et à Suzanne.- C'est un billet doux, qu'une fillette aura glissé dans sa main en passant. Il était cacheté d'une épingle, qui l'a outrageusement piqué. (Beaumarchais, Le mariage de Figaro, acte IV, Scène IX)

(9a) S'il est cacheté d'une épingle, c'est un billet doux (si p, q)

Or il est cacheté d'une épingle (p)

Donc c'est un billet doux (q)

(q) est la conclusion d'un syllogisme déductif.

(9b) Si c'est un billet doux alors il est d'une femme (si p, q)

Or (je sais que) c'est un billet doux (étant donné 9a) (p)

Donc c'est une femme (q)

Une représentation cohérente de la situation se dessine. Le locuteur procède à l'élaboration d'une histoire rassemblant les différents indices en se fondant d'une part, sur des connaissances communes (ou encyclopédiques) et d'autre part sur les connaissances d'arrière-plan liées au contexte. Nous changeons de type de raisonnement lorsque Figaro précise la démarche entreprise par la fillette. Le locuteur suppose la proposition comme vraie et la présente donc au futur conjectural.

(9c) Le billet est entre les mains du Comte (q)

Si une fillette a glissé un billet dans la main du Comte alors le Comte a un billet entre les mains. (Si p, q)

Une fillette aura glissé le billet dans sa main en passant (p).

Paraphrase : c'est une fillette qui a glissé le billet dans sa main en passant mais cela reste à vérifier.

19 En conséquence, (p) se présente comme une explication plausible, fondée sur une connaissance commune (ou règle générale, d'après Desclés \& Guentchéva, 2001) qui prendrait la forme suivante: Si une fillette a glissé un billet dans la main du Comte alors le Comte a un billet dans la main. La différence entre les deux phrases suivantes réside donc dans l'obtention de la valeur de vérité.

(10a) C'est un billet doux qu'une fillette a glissé dans sa main.

(10b) C'est un billet doux qu'une fillette aura glissé dans sa main.

En (10a), l'absence du futur conjectural supprime toute distance de la part du locuteur, la proposition est assertée tandis que le locuteur de l'exemple (10b) asserte la plausibilité/ probabilité de la conclusion. La valeur de plausibilité/probabilité ne provient pas du fait que les prémisses véhiculent de l'incertitude. On peut très bien paraphraser la phrase par :

(10c) C'est un billet doux qu'une fillette aura très certainement glissé dans sa main.

21 Auquel cas, le futur conjectural serait incompatible avec des expressions modales exprimant la certitude. Ce qui nous amène à la conclusion suivante:le processus inférentiel engendre une valeur de probabilité/plausibilité. Celle-ci indique que la proposition ne peut être assertée mais n'implique pas pour autant que les prémisses véhiculent de l'incertitude. Le fait est supposé vrai, ce qui permet au futur conjectural d'être substituable par un passé composé. La paraphrase suivante reste acceptable sans trop modifier le sens d'origine :

(10d) C'est un billet doux qu'une fillette a glissé dans sa main, cependant mon propos reste à vérifier.

22 Nous pouvons ainsi en conclure qu'un énoncé au futur conjectural est le produit d'un processus inférentiel mis en œuvre à partir d'une ou plusieurs prémisses présentes dans le contexte. Le futur conjectural porterait sur une irrévocabilité rétrospective puisque l'assertion est antérieure au moment où elle est avérée. Il ne porte donc pas sur la proposition. 


\section{La valeur du futur conjectural}

selon ces trois paramètres : le mode personnel-actuel, le temps futur/futur antérieur et l'aspect global/global extensif. Selon Wilmet, la variable temps futur suffit à engendrer les principaux emplois répertoriés ; l'effet conjectural est " plutôt redevable à l'aspect global » (Wilmet, 1987 : § 483, 378). Pour notre part, nous sommes amenées à penser que ce futur conjectural conserve sa valeur temporelle de futur, la présence de l'une des deux valeurs n'impliquant pas nécessairement l'exclusion de l'autre. Vetters (2001) compare le futur des historiens au futur conjectural. La temporalité objective est présente dans le cas du futur simple ou passée dans le cas du futur antérieur. De plus, l'emploi du futur conjectural est caractérisé en termes de temporalité de dicto (Martin, 1987). La notion temporelle d'avenir est présente puisque la possibilité de confirmer le fait est ultérieure, «seul l'avenir confirmera épistémiquement, ce qui au présent n'est qu'une hypothèse vraisemblable », (Martin, $1987: 117$ ).

28

Quelle est la valeur en français de ce futur dit conjectural, considéré par la tradition comme résolument modal ? Cette question s'inscrit dans une problématique plus générale concernant la dichotomie entre modalité et temporalité faite par les linguistes : ce qui est considéré comme temporel ne pourrait être conçu comme modal et réciproquement. Gosselin (à paraître) remet en question cette conception traditionnelle des relations entre temporalité et modalité. Il considère que cette classification repose sur un découpage entre le réel et l'irréel, assimilant le réel au certain (le temporel) et l'irréel à l'incertain (le modal). Un événement ne pourrait être conçu à la fois comme réel et irréel ; ce qui est possible ou incertain, voire impossible se localise aussi dans le temps comme événement passé, présent ou futur.

véhiculer directement une valeur temporelle. Vet \& Kampers-Manhe (2001: 95) signalent que le futur simple ou « forme en - $r a$ » fonctionne dans la tournure conjectural comme un opérateur modal et non comme un opérateur temporel. Le futur, dans sa tournure conjecturale, marquerait une certaine distance quant à la valeur de vérité véhiculée par la proposition.

supposition comme un des emplois modaux du futur simple/antérieur :

Quand un procès contemporain de l'énonciation n'est pas avéré, le futur simple est employé pour le présenter comme une hypothèse, que l'avenir confirmera ou permettra de vérifier (...). L'énoncé au futur simple est souvent associé à une phrase au présent ou au passé à laquelle il apporte une explication possible, car reportée dans l'avenir (...). (1994: 314-315)

Une correspondance est établie entre la valeur temporelle du futur antérieur et celle du passé composé : Elle revient déjà : elle aura manqué son train équivaut à elle a manqué son train . « Il [le futur antérieur] projette fictivement dans l'avenir une hypothèse probable, que celui-ci devra confirmer » $(1994: 315)$.

En berbère kabyle, le problème est similaire. Considérée comme une langue à « aspects ", le berbère kabyle possède quatre thèmes verbaux : l'aoriste simple, [ad + aoriste], l'aoriste intensif et le prétérit, tous allèguent une valeur aspectuelle aux propositions. La valeur 
aspectuo-temporelle de ad combiné au thème de l'aoriste n'a jamais, à notre connaissance, été déterminée. Néanmoins, il est mentionné, dans une liste non exhaustive, les différents contextes dans lesquelles cette particule peut apparaitre ainsi que ses valeurs (entre autres, Chaker, 1997, Mettouchi, 2002).

La particule ad soulève de nombreuses polémiques parmi les linguistes berbérisants. Les travaux de A. Hanoteau (réed. 1982) et plus tard ceux de Dallet (1960) ont identifié la particule ad comme exprimant un futur. Cette notion de temps a été rejetée par un grand nombre de linguistes. Chaker note que la «notion de temps » n'est pas exprimée par les thèmes verbaux; le contexte ou une particule jointe au verbe, ou un adverbe de temps indiquera si la proposition est au présent, au passé ou au futur. Certains estiment qu'elle marque l'aspect dont la valeur est de signifier une action "inaccompli, irréelle ou indéfini » (entre autres, Chaker, 1983/1984, Penchoen, 1973). D'autres, lui attribuent une valeur modale et établissent une opposition entre un mode réel (prétérit) et un mode non réel (ad + aoriste) (Bentolila, 1981, Leguil, 1987, Galand, 1987). Sadiqi ${ }^{7}$ remarque que l'expression temporelle dans les textes berbères ne peut être que la somme de valeurs exprimées par la conjugaison verbale et tous les éléments composants le contexte bien que le système verbal soit d'abord aspectuel. Il nous semble que chacun de ces points de vue divergents met en avant une des instructions de la combinatoire. En considérant la particule ad comme polysémique, nous verrons que sa valeur aspectuo-temporo-modale dépend en grande partie du contexte. Nous montrerons que ce marqueur code une ou plusieurs instructions pour la représentation du procès sur l'axe temporel. Dans le cadre d'un article, nous ne pouvons pas prendre en compte tous les effets de sens de la construction préverbale [ad + aoriste]. Nous focaliserons notre analyse sur la valeur modale exprimée dans les contextes A (cf. Le rôle du contexte).

\section{Le modele calculatoire et cognitif du temps et de l'aspect}

Gosselin $(1996,2000)^{8}$ propose un modèle calculatoire et cognitif du temps et de l'aspect qui permet de rendre compte des effets de sens en contexte généralement constitutif de la polysémie des éléments de l'énoncé ${ }^{9}$ et s'inscrit dans une démarche hypothéticodéductive. Nous ne présenterons pas le modèle général sur le temps, l'aspect et les modalités dans toute sa complexité mais uniquement les paramètres nécessaires à notre analyse. Dans ce modèle, les marqueurs linguistiques (lexèmes, morphèmes grammaticaux et constructions syntaxiques) codent une ou plusieurs instructions pour construire des représentations aspectuo-temporelles. La signification se construit donc en contexte. Ces instructions peuvent entrer en conflit. Ces conflits (intralinguistiques ou pragmatico-linguistiques) sont résolus au moyen de déformations régulières qui peuvent être prédictibles.

31 Le modèle utilisé propose une représentation aspectuo-temporelle à partir de quatre intervalles disposés sur l'axe du temps qui entretiennent des relations entre eux. L'intervalle de référence, noté [I, II], correspond à ce qui est montré/perçu du procès (une scène) à un moment donné du temps. L'intervalle de procès [B1, B2] détermine une situation telle qu'elle est catégorisée par le prédicat. L'intervalle d'énonciation [01, 02] correspond à l'énonciation, en fonction de la prise de parole. L'intervalle circonstanciel [Ct1, Ct2] est facultatif et correspond aux circonstanciels de localisation. Sa portée varie 
en fonction de la construction syntaxique de la phrase : sur l'intervalle de référence ou sur celui du procès. Les relations entre intervalles permettent le calcul de ces représentions.

L'aspect grammatical est considéré comme le point de vue interne du procès; on peut voir son déroulement interne de façon globale (aoristique) ou dans ses phases successives (la phase préparatoire ou résultante). Dans ce modèle, l'aspect grammatical est défini par la relation entre l'intervalle de procès et l'intervalle de référence :

- Aspect aoristique : [I, II] coïncide (CO) avec [B1, B2] : le procès est vu de façon globale (Il prit le train de $19 \mathrm{~h} 20$ pour Paris.)

- Aspect inaccompli : [I, II] est recouvert par (RE) [B1, B2] : le procès est perçu/montré dans son déroulement. (Jean se promenait depuis dix minutes quand il croisa Marie)

- Aspect accompli : [I, II] est postérieur (POST) à [B1, B2] : seul l'état résultant du procès est saillant. (Il est parti voilà dix minutes)

- Aspect prospectif : [I, II] est antérieur (ANT) à [B1, B2] : la phase préparatoire du procès est envisagée. (Attention, il va tomber)

L'hypothèse postule que les relations entre l'intervalle d'énonciation et l'intervalle de procès ne sont pas directement contraintes. Le temps absolu correspond à la relation entre l'intervalle de référence et celui de l'énonciation :

- Passé: [I, II] ANT [01, 02]

- Présent: [I, II] CO [01, 02]

- Futur: [I, II] POST [01, 02]

La valeur en langue du futur est définie hors contexte, elle est donc stable et permet de prédire les effets de sens. En français, le futur ne code qu'une instruction de nature temporelle :

- Un temps absolu : futur [I, II] POST [01, 02]

L'effet de sens typique du futur est applicable au principe de dépendance contextuelle de l'intervalle de référence, celui-ci étant anaphorique, il doit se rattacher à un élément pour permettre son ancrage. L'intervalle de référence [I, II] se trouve généralement lié à $[\mathrm{B} 1, \mathrm{~B} 2]$ avec lequel il coïncide, ce qui confère au procès un aspect aoristique :

- Un aspect : aoristique [I, II] CO [B1, B2]

Le classement des modalités se fait selon quatre niveaux. Nous nous intéresserons au quatrième niveau, celui des modalités temporelles et aspectuelles. Ce niveau est important pour comprendre la suite de notre analyse. Le domaine modal se découpe en deux champs : l'irrévocable et le possible. L'hypothèse linguistique avancée par Gosselin est que la coupure modale porte sur la borne finale de l'intervalle d'énonciation lorsqu'il s'agit de considérer le point de vue des modalités temporelles. En revanche, les modalités aspectuelles sont considérées à partir du point de vue de ce qui est perçu du procès, à savoir son moment de référence; la coupure modale portera donc sur la borne finale de l'intervalle de référence, déterminant ainsi le champ de l'irrévocable de celui du possible.

La coupure modale porte sur la borne finale de l'intervalle d'énonciation, on obtient les modalités temporelles. 
Figure $n^{\circ} 1$

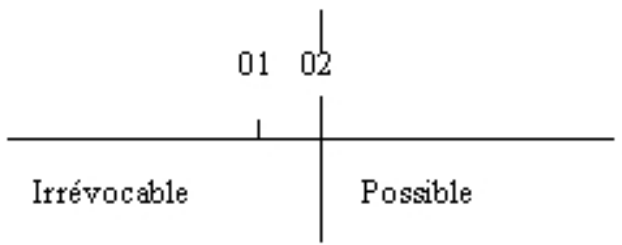

37 Hypothèse linguistique : la coupure modale porte sur la borne finale de l'intervalle de référence [I, II], on obtient les modalités aspectuelles.

Figure $n^{\circ} 2$

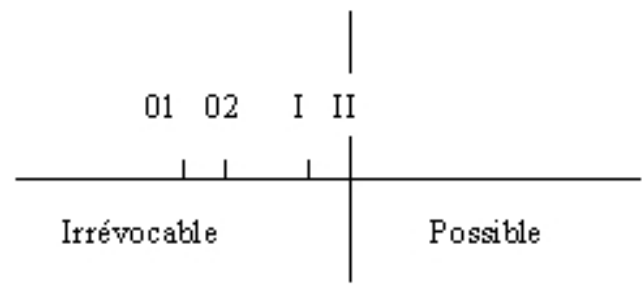

38 Si l'on applique cette coupure modale aux quatre aspects de la langue française, nous pouvons prédire les effets de sens suivants :

Figure $n^{\circ} 3$

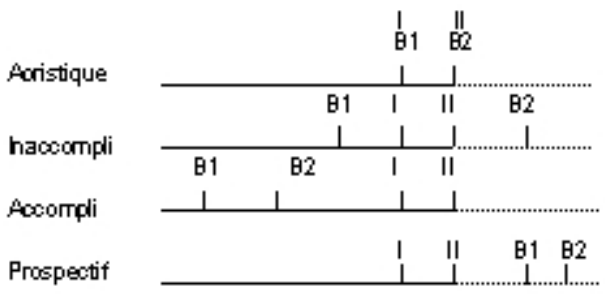

39 Ainsi, les modalités temporelles situent le futur dans le champ du possible tandis que les modalités aspectuelles le placent dans le champ de l'irrévocable; ce qui engendre un conflit. Celui-ci est résolu par la dimension épistémique : la certitude subjective ou visée intentionnelle. 
Figure $n^{\circ} 4$

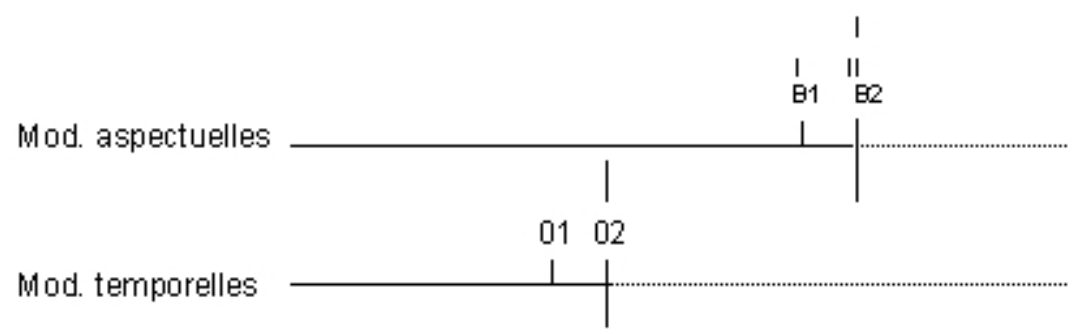

En nous appuyant sur l'analyse proposée par Gosselin (1999) pour les systèmes hypothétiques, nous essaierons de décrire les instructions liées à [ad + ili] + prétérit/ aoriste intensif.

\section{Les trois types de fonctionnement de la modalité linguistique}

Gosselin propose une analyse reposant sur les concepts de méta-prédicat et par conséquent de méta-procès. Il rappelle les deux grands types de distinction établis pour définir les modalités :

(a) l'opposition entre modalité de re et modalité de dicto reprise par Quine (1966),

dans une approche logique, en termes de «quantification within» et

« quantification into a modal context »;

(b) les modalités véridicibles/non véridicibles de Kronning (1996).

Les modalités véridicibles sont susceptibles d'être niées ou interrogées. Bien que les modalités de re soient toujours véridicibles, Vet (1997) a montré que les modalités de dicto pouvaient être véridicibles ou non. Gosselin propose de reconsidérer les modalités en partant de l'analyse faite par Quine (1966); les trois types de fonctionnement des modalités linguistiques correspondent aux « trois degré d'engagement modal » distingués par Quine :

(c) « opérateur prédicatif » (modalité de re véridicible)

(d) « opérateur propositionnel » (modalité de dicto non véridicible)

(e) « prédicat sémantique » (modalité de dicto véridicible).

Il envisage donc la modalité de dicto véridicible comme méta-prédicat. Cette dernière porterait sur le contenu sémantique de la proposition. L'hypothèse linguistique avancée par Gosselin part du postulat suivant: le méta-prédicat exprime un méta-procès (un procès qui affecte un autre procès). L'exemple cité (1999 : 46), « il est probable que Paul va venir » véhicule une proposition affirmant le premier procès « la venue de Paul » comme probable par le deuxième procès. En s'inscrivant dans son modèle du temps et de la l'aspect, tout procès se situe dans la temps et sous un certain aspect. Le méta-prédicat porte sur un méta-procès, il exprime donc une temporalité et un aspect qui lui sont propres, indépendamment du moment de l'énonciation, à la différence des opérateurs propositionnels. 


\section{L'analyse contrastive} primaires. Le thème verbal exprime un aspect qui s'inscrit, selon notre analyse, dans le temps ${ }^{10}$. Traditionnellement, l'accompli et l'inaccompli sont considérés comme les deux valeurs marquant l'opposition aspectuelle, l'aoriste simple est caractérisé comme marqueur modal dans la mesure où cette forme n'est pas autonome (Galand, 1987 ; Leguil, 1987). La valeur de [ad + aoriste] demeure problématique. Nous avons donc deux valeurs aspectuelles pour quatre formes :

(f) Aoriste simple : A y-yemnec Rebbi, yejber-ay!

(Que Dieu nous épargne et nous préserve!)

(g) Aoriste intensif : yeznuzuy irden

(Il vend/vendait du maïs)

(h) [ad + aoriste] : ad-yečč

(Il mangera/va manger)

(i) Prétérit ou prétérit négatif ččiy / ur ččiy ara

(J'ai mangé)

nous intéresserons plus particulièrement à la forme [ad + aoriste] dans le type de contexte comme défini en deuxième partie. [Ad + aoriste] est incompatible avec un circonstanciel de localisation temporel exprimant un passé :

*(11) Idelli ad yeddu yid es.

*Hier, il partira avec lui.

Il n'est pas compatible avec un circonstanciel de localisation temporel exprimant un présent sans en changer sa valeur aspectuelle :

*(12) Tura ad yeddu yid es.

(Maintenant), il va partir avec lui.

En nous inscrivant dans le modèle de Gosselin, le thème [ad + aoriste] code les instructions suivantes:

Aspect aoristique: [I, II] CO [B1, B2]

Temps présent : [I, II] POST [01, 02]

Munis de ces valeurs en langue, nous tenterons de donner les instructions codées par [(ad + aoriste) + prétérit/aoriste intensif] où celui-ci a une valeur conjectural. La supposition ou l'antériorité dans le futur est représentée par la même forme; les informations contextuelles permettent d'opter pour l'une ou l'autre de ces valeurs. Autrement dit, les valeurs véhiculées par les thèmes verbaux dépendent du contexte. Il faudra tenir compte de deux facteurs qui déterminent l'effet de sens de conjecture dans les constructions en [ad + ili (aoriste)]. Examinons le rôle du contexte :

(13) Ad iliy ruhey Je serai (déjà) sorti.

(14) Ad yiky yeffey

Il sera sorti.

(15) Ad nik nečča (yagi)

Nous aurons (alors) mangé.

Les exemples (13) et (15) ne peuvent recevoir une interprétation inférentielle exprimant une vérification future. Les pronoms personnels de première personne du singulier et du pluriel imposent une prise en charge du discours; un contexte d'ignorance s'impose donc.

[Ad + ili (aoriste)] + Prétérit : le procès est vu comme accompli. 
(16) Ad yili yečča

(16a) Il aura mangé (étant donné l'heure à laquelle il vaarriver).

(16b) Il aura mangé.

[Ad + aoriste $]+$ Aoriste intensif : le procès est vu comme inaccompli.

(17) Ad yili yeŢ̧̦e

(17a) Il sera en train de manger (puisqu'il n'est toujours pas là).

(17b) Il sera en train de manger le couscous.

ans les exemples (16a) et (17a), ces propositions peuvent recevoir une interprétation inférentielle exprimant la probabilité/plausibilité que le fait supposé soit vrai sous réserve d'une vérification future. Dans les exemples (16b) et (17b), la valeur de supposition est remplacée par la certitude épistémique. La valeur de conjecture est le résultat d'un processus inférentiel fondé sur des faits observés dans le présent du locuteur. Ce deuxième facteur nous permet de retenir la valeur de vérification future dans les tournures conjecturales. Lorsque ces deux facteurs sont présents le futur prend une valeur conjecturale auquel cas, la valeur de certitude épistémique s'applique par défaut.

Nous partons de l'hypothèse suivante :

Le futur conjectural ne réfère pas à la proposition mais porte sur la vérification exprimée par le second procès. Celui-ci est considéré comme un méta-procès, à savoir un procès qui caractérise l'état du premier procès et correspond à l'irrévocabilité rétrospective que $\mathrm{p}$ soit vrai. Le procès exprimé par la proposition sera antérieur au méta-procès.

(18) (A) Ad yili d tidett (B) yečča

Il aura été vrai qu'il a mangé.

(19) (A) Ad yili d tidett (B) yetta.

Il aura été vrai qu'il a été en train de manger.

La modalité exprimée par le futur conjectural (18) et (19) porte sur le procès de la proposition (B), celui-ci est asserté rétrospectivement. De plus, la coupure modale portant sur la borne finale de l'intervalle de référence du premier procès, le futur situe cette irrévocabilité rétrospective dans le champ du possible. Le procès est antérieur à cette irrévocabilité puisque celle-ci est considérée comme rétrospective.

A partir des instructions codées par [ad + ili], nous proposons la représentation suivante :

Figure $n^{\circ} 5$

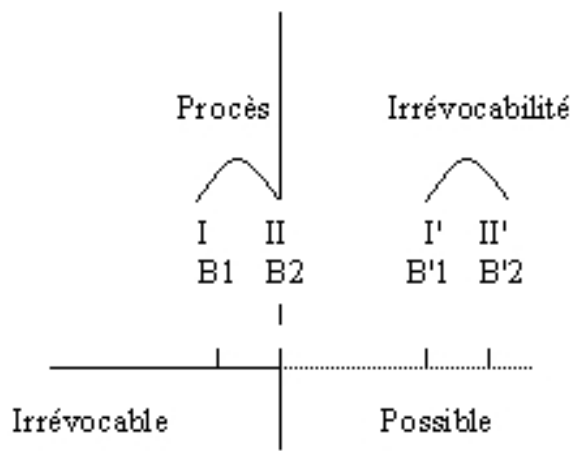

Corela, HS-1 | 2005 


\section{8. conclusion}

51 Dans un premier temps, nous avons montré l'importance du contexte pour distinguer les différentes valeurs des emplois de [ad + ili(aoriste) + prétérit/aoriste intensif]. Nous avons démontré que la valeur de probabilité/plausibilité exprimée dans un énoncé au futur conjectural ne provenait pas de l'incertitude des prémisses mais du processus inférentiel mis en œuvre. L'hypothèse défendue ici est que [ad + ili] porte sur la vérification, celle-ci est considérée comme un méta-prédicat (un procès qui porte sur un autre procès) correspondant à une irrévocabilité rétrospective fondée sur les relations qui unissent l'aspect et la modalité (modalités aspectuelles). Dans le cadre d'un modèle hypothéticodéductif, les marqueurs linguistiques codent des instructions qui permettent de prédire les effets de sens en contexte de [ad + ili (aoriste) + prétérit/aoriste intensif], en particulier, le cas de la conjecture. En effet, dans le cadre d'un article, il n'est pas envisageable de prendre en considération la totalité des paramètres sémantiques. Nous avons tenté de montrer que la construction en [ad + ili] est à même d'exprimer une valeur temporelle en rendant compte de la dimension modale véhiculée par l'énoncé.

\section{BIBLIOGRAPHIE}

BENTOLILA, F. (1981). Grammaire fonctionnelle d'un parler berbère : Aït Seghrouchen d'Oum Jeniba (Maroc), Paris : SELAF

BORILLO, A. (2001). « La modalité épistémique : le cas du futur conjectural ». Communication au colloque, Temps et Point de vue. Université de Paris III

CHAKER, S. (1985). « Notice "AD (grammaire/verbe) ». Encyclopédie Berbère II. Aix-en-Provence : Edisud. 115-116

CHAKER, S. (1997). «Quelques faits de grammaticalisation dans le système verbal berbère », Mémoire de la Société Linguistique, Nouvelle Série n5, Louvain : Peeters, 103-121

DAMOURETTE, J. \& PICHON, E. (1911-1936). Des mots à la pensée. Essaie de grammaire de la langue française tome 5. Paris : d'Artrey

DALLET, J.-M. et Sr LOUIS de VINCENNES. (1960). Initiation à la langue berbère. Grande Kabylie : Fort National

DENDALE, P. (2001). « Le futur conjectural versus devoir épistémique : différences de valeur et restrictions d'emploi ». Le français moderne $\mathrm{n}^{\circ} 1$ 1-20

DENDALE, P. (1994). « Devoir épistémique, valeur modale ou évidentielle? ». Langue française n - 102. 24-40

DENDALE, P. \& De MULDER, W. (1996). « Déduction ou abduction : le cas de devoir inférentiel ». GUENCHÉVA, Z. (éds.). L'Enonciation médiatisée. Paris/Louvain : Peeters. 305-318 
DESCLÉS, J.-P. \& GUENTCHÉVA, Z. (2001). « La notion d'abduction et le verbe devoir “épistémique'« . Cahiers Chronos n 8. Amsterdam : Rodopi. 103-122

DESCLÉS, J.-P. (1999). « Au sujet de la catégorisation verbale ». Faits de langue nº 14. 227-239

DESCLÉS, J.-P. (1997). « L'abduction, procédé d'explication en linguistique ». Modèles linguistiques n -34. XVII : 2

ECO, U. (1990). Les Limites de l'interprétation. Paris : Grasset

GALAND, L. (1987). « Les emplois de l'aoriste sans particule en berbère ». Proceedings of the Fourth International Hamito-Semitic Congress. Amsterdam: John Benjamins Publishing Company. 361-380

GÄRDENFORS, P. (1988). Knowledge in Flux: Modelling the Dynamics of Epistemic States. Cambridge: Bradford Books, MIT Press

GOSSELIN, L. (à paraître). Observations linguistiques sur l'irréversibilité du temps

GOSSELIN, L. (2001). « Relations temporelles et modales dans le conditionnel journalistique ». Le conditionnel en français. DENDALE, P., TASMOWSKI, L. (éds.). Université de Metz. 45-66

GOSSELIN, L. (2000). « Le statut du temps et de l'aspect dans la structure modale de l'énoncé.

Esquisse d'un modèle global ». Syntaxe \& Sémantique $\mathrm{n}^{\circ} 2.57-80$

GOSSELIN, L. (1999). « Les valeurs de l'imparfait et du conditionnel dans les systèmes

hypothétiques ». Cahiers Chronos, $\mathrm{n}^{\circ} 4.29-52$

GOSSELIN, L. (1998). « Le paradoxe imperfectif ou la disjonction entre assertion et prédication ». FORSGREN, M., JONASSON, K., KRONNING, H. (éds). Prédication, assertion, information. Université d'Uppsala : Acta Universitatis Upsaliensis. 211-219

GOSSELIN, L. (1996). Sémantique de la temporalité en français. Un modèle calculatoire et cognitif du temps et de l'aspect. Louvain-la-Neuve : Duculot

HANOTEAU, A. (1982). Essai de grammaire de la langue Tamachek. Paris: Réed. POF-Inalco

KLEIN, W. (1994). Time in Language. Londres: Routledge

KRONNING, H. (1996). Modalité, cognition et polysémie : sémantique du verbe « modale » devoir. Stockholm : Acta Universitatis Upsaliensis

LEGUIL, A. (1987). Structure prédicative en berbère. Doctorat d'État sous la direction de David Cohen. Université de Paris III

MARTIN, R. (1987). Langage et croyance. Bruxelles : Margada

MARTIN, R. (1991). « Types de procès et systèmes typologique ; de l'aspect 'de re' à l'aspect 'de dicto' " . Travaux de linguistique et de philosophie $\mathrm{n}^{\circ} 22$. 87-95

METTOUCHI, A. (1998). « Aspect et négation : remarques sur l'inaccompli et la négation en anglais et en berbère (kabyle) ». Cahiers Chronos, $n^{\circ} 2.191-205$

METTOUCHI, A. (2002). « La forme ad + aoriste en berbère (kabyle) » in NAÏT-ZERRAD, K. (éd.) Articles de linguistique berbère. Mémorial Werner Vycichl, Paris : L'Harmattan, 335-347

NAÏT-ZERRAD, K. (2001). Grammaire moderne du kabyle - tajerrumt tatrart $\mathrm{n}$ teqbaylit. Paris : Karthala

PEIRCE, C.S. (1955). Abduction and Induction. Philosophical writings of Peirce. NY : Dover PENCHOEN, Th. 1966/1973, Étude syntaxique du parler berbère (Chaouïa) des Aït Frah (Aurès). D'après les textes d'Alain Basset. Thèse pour le Doctorat de 3ème cycle. Paris 
QUINE, W.v.O. (1966). The Way of Paradox and Other Essays. Cambridge: Harvard U.P

REICHENBACH, H. G. (1947). Elements of Symbolic Logic. NY: Dover

RIEGEL, M., PELLAT, J.-C. \& RIOUL, R. (1994). Grammaire méthodique du français. Paris : Presses Universitaires de France

SCHÄFER-PRIEß, B. (2001). « Le futur de probabilité ». Revue de Sémantique et Pragmatique n 9/10. $9-17$

VET, C. \& KAMPERS-MANHE, B. (2001). « Futur simple et futur du passé : leurs emplois temporels et modaux ». Le conditionnel en français. DENDALE, P., TASMOWSKI, L. (éds). Université de Metz. 89-104

VET, C. (1993). « Conditions d'emploi et interprétation des temps futurs en français ». Verbum $\mathrm{n}$ $\circ$ 4. Université de Nancy II. 71-84

WILMET, M. (1997). Grammaire critique du français. Louvain-la-Neuve : Duculot

WILMET, M. (1997). « L'articulation mode-temps-aspect dans le système français ». Temps et langage (I). Actes du colloque international tenu à Paris-Sorbonne. Lille : ENSAM. 91-110

\section{NOTES}

1. Je tiens à remercier L. Gosselin pour ses critiques et commentaires constructifs.

2. Corpus d'énoncés bilingues extraits de Genevoix, H., réed. 1996, Villages de Kabylie, ENAG, Alger. La notation de cette édition s'aligne sur la notation d'inspiration phonologique telle qu'elle est recommandée par S. Chaker.

3. Dendale note que Chevalier mentionne un exemple de futur conjectural autre que les verbes susdits : Notre ami est absent. Il présidera quelque réunion. (1978: 352)

4. Naït-Zerrad rend compte de la dimension modale dans les traductions françaises en caractérisant la probabilité par devoir épistémique.

5. Comme le souligne Dendale (2001: 10), cette notion évoquée par Schrott n'est pas très explicite. Les paramètres qui permettent de délimiter le monde des expériences ne sont pas évoqués.

6. Ici, l'ignorance est définie comme un état de non savoir indépendamment du fait que la personne puisse mentir (cf. l'exemple (1)) .

7. Sadiqi, F., Grammaire berbère, L'Harmattan, Paris, 1997

8. Un modèle similaire a été développé par Klein (1994) pour l'anglais

9. Gosselin décrit en termes de polysémie contextuelle générale le fait que la signification d'un marqueur puisse varier en fonction de ses formes et de la signification des autres marqueurs en contexte.

10. Bellahsène, L. (à paraître). Analyse contrastive français/berbère d'un marqueur aspectuotemporel : le cas de la particule ad. 


\section{RÉSUMÉS}

Cet article présente une analyse contrastive français/berbère (kabyle) du futur antérieur et de la particule [ad + ili(aoriste)]. Nous étudierons un point particulier, celui de l'expression de la conjecture comme modalité liée directement au traitement de la temporalité et de l'aspectualité. La conception aristotélicienne donne une interprétation temporelle de la modalité : le possible est passé, présent ou futur. Dans son modèle, L. Gosselin propose une définition aspectuelle de la modalité. Partant, de cette définition, nous défendrons l'hypothèse selon laquelle nous considérons les marqueurs linguistiques comme des unités polysémiques permettant l'expression du temps et de la modalité véhiculée par la proposition au moyen de combinatoires complexes. L'absence de marques morphologiques dédiées uniquement à l'expression de la temporalité dans la langue berbère (kabyle) n'implique pas pour autant, qu'elle n'est pas à même de rendre compte du découpage temporel imposé par le texte et d'exprimer la modalité véhiculée par la proposition (par exemple, lors d'une traduction).

This paper presents a contrastive analysis French/Berber (Kabylian) of the future perfect and the particle [ad + ili (aorist)]. We will focus on a particular point. The relation of modality to time in Aristotle's view is that all genuine possibilities are actualized in time. Thus, any possibility has been, is or will be actualized. L. Gosselin proposes a definition on what the relation of modality to aspectuality should constitute. In view of this definition, we will argue that time or modality value does not only result from languages which encode distinction by means of grammatical elements (inflectional morphemes). In traditional linguistic analysis, the verbal system of Berber (Kabyle) encodes only aspectual oppositions. However, one expresses time as one translates a text in French. I submit that linguistic elements of sentences are polysemous and can express time and modality which is the result of a complex combinatory of units.

\section{INDEX}

Mots-clés : sémantique, cognition, aspect, temps, modalité, polysémie, langue berbère kabyle.

\section{AUTEUR}

\section{LINDA BELLAHSÈNE}

Université de Rouen 